\title{
Development and Psychometric Properties of TQWL-42 to Measure the Quality of Work Life
}

Bruno Pedroso ${ }^{1^{*}}$

https://orcid.org/0000-0002-7905-2393

Luiz Alberto Pilatti ${ }^{2}$

http://orcid.org/0000-0003-2679-9191

Gustavo Luis Gutierrez ${ }^{3}$

http://orcid.org/0000-0002-2383-8696

Claudia Tania Picinin ${ }^{2}$

http://orcid.org/0000-0003-4844-3516

${ }^{1}$ Universidade Estadual de Ponta Grossa; ${ }^{2}$ Universidade Tecnológica Federal do Paraná;

3 Universidade Estadual de Campinas

Received: 2018.07.20; Accepted: 2019.03.18.

HIGHLIGHTS

- Most QWL's models is grounded in classical theoretical models.

- These models is obsolete and based on the US culture.

- There is no instruments to assess the QWL as a whole or globally.

- TQWL-42 was based on the lifestyles of contemporary society.

Abstract: The objective of this study was to construct and validate an instrument that evaluates the Quality of Work Life (QWL) globally, following the WHOQOL instruments and founded in QWL's classical theoretical models, directed to the contemporary society. The psychometric properties tested were content validity, face validity, internal consistency, criterion validity, concurrent validity and test-retest reliability in an application to 283 individuals. The final version of the instrument is constituted by 47 questions, being five to sample knowledge and 42 divided in five spheres that contemplate QWL's dimensions. For the results calculation a SPSS syntax and a tool in Microsoft Excel that perform the automated calculation after the data tabulation were developed. The psychometric properties tested were satisfactory. We conclude that the goal of validating a global instrument for evaluation of QWL from the contemporary society culture with satisfactory psychometrical characteristics was reached.

Keywords: Quality of work life; Measurement instrument; Psychometric properties. 


\section{INTRODUCTION}

The concern for Quality of Life (QOL) has increased in recent decades. The holistic view of human as a bio-psycho-social being is gaining space, including in the business environment. This reflection is strengthened from the perception that workers' performance is strongly related to their QOL.

During the 1970's, several instruments for evaluating the QOL appeared. However, such instruments do not assess QOL per se, but rather the Health-related quality of life (HRQOL). Facing this scenario, the World Health Organization (WHO) was committed to creating an global instrument for the assessment of QOL, in which all the aspects related to QOL are listed, transcending the HRQL. As a consequence, the WHOQOL Group arises in 1993 [1].

Successive labor demonstrations that occurred throughout the twentieth century prompted researchers from different fields to investigate the phenomenon of QOL in the work environment, which subsequently breaks down from its progenitor variable, giving rise to a new indicator: the Quality of Work Life (QWL) [2].

With the advent of the industrial revolution, work began to be humanized. The subhuman conditions, under which the work was conducted at that time, made the workers to protest for higher wages, lower working hours and better working conditions. Concern about the health and wellbeing of the worker has since then been intensified. However, even if the productive environment has undergone a revolution, the improvement of QWL has been restricted. In an attempt to measure this variable, different theoretical models were outlined [2].

Despite the existence of measurement instruments structured from theoretical models for assessing the QWL, it is pertinent to note that most of these instruments is grounded in QWL's classical theoretical models [3-7]. Although widely used and referenced in the Brazilian literature, such models have been built over more than two decades and were also developed and validated based on the US labor culture.

Therefore, it is evident the lack of instruments to assess the QWL as a whole, or globally, as occurs with the assessment of QOL by WHOQOL-100 and WHOQOL-bref [1].

In this perspective, the objective of this study is to construct and validate an instrument to assess globally the QWL, following the patterns of WHOQOL instruments (whose psychometric properties are globally accepted), and grounded in QWL's classical theoretical models, with direction to the Brazilian contemporary society.

\section{MATERIAL AND METHODS}

The process of construction and validation of TQWL-42 instrument consisted of the following steps: a) Literature review; b) Selection of QWL's indicators to compose the instrument; c) Instrument construction; d) Pilot test; e) Content validity; f) Face validity; g) Field test.

In the end of the stages of literature review, we follow the WHOQOL methodology [1] for selection of QWL's indicators to compose the instrument (based on the analysis of the QWL's classical theoretical models which underpin the present instrument), and for design an instrument pilot, which was tested in a group of 26 employees of a multinational industry in the branch of chemistry, located in the city of Ponta Grossa (Paraná, Brazil). The plan of analyzing these data aimed to select to the final version of the instrument the best questions for each dimension of the instrument.

A test of content validity of the instrument was also carried out, which was evaluated by a group of judges consisting of six PhD researchers in the QWL area. The requested changes were examined and followed. Finally, the instrument was subjected to the evaluation of a professional of linguistics to the conference of spelling and appropriateness of the terms used to the face validity of the instrument.

The analysis procedures of the final version consisted of the application of TQWL-42 in a sample of 283 workers from various organizations of different sizes on the city of Ponta 
Grossa (Paraná, Brazil), in order to measure its psychometric properties concerning the criterion validity, using the Pearson product-moment correlation coefficient between the spheres of the instrument and internal consistency by Cronbach's alpha. To verify the normality of the data we used Kolmogorov-Smirnov's test.

To the concurrent validity, the TQWL-42 application was performed together with the Walton's QWL instrument, whose Brazilian version was validated by Timossi et al. [8], and the Pearson product-moment correlation between the total score of TQWL-42 and the overall score of the Walton's QWL instrument.

Once 14 days have elapsed from the first application, the TQWL-42 was reapplied to 76 workers who participated in the first application for the assessment of test-retest reliability, analyzing the variability of mean scores of the spheres of instrument between both applications and checking the Pearson product-moment correlation of mean scores of the spheres between test and retest.

Regarding ethical issues, the conduction of this study was approved by the Research Board and Postgraduate of Federal University of Technology - Paraná (UTFPR) Campus Ponta Grossa, under Official Notice № 01/2011. All employees who participated in the pilot test and final application of the instrument object of this study signed a consent form on the objectives and purposes of the research.

To calculate the scores of the instrument a syntax using SPSS software was constructed, whose use is suggested by the WHO to calculate the scores of WHOQOL instruments.

Given the difficulty in using the SPSS software and the fact that most Brazilian institutions do not have the registration that legalizes the use of such software, a tool on Microsoft Excel software was also built, such as the tool designed by Pedroso, Pilatti and Reis [9], which performs the calculation of scores and descriptive statistics of the instrument developed in this study.

In the tool, all calculations and graphs are performed automatically. The researcher must simply tabulates the collected data in the specified locations. The use of Microsoft Excel is justified by the fact that this software is globally widespread and used by researchers from all areas of knowledge.

The tool was built from the software Microsoft Excel 2003, also being tested in versions $2000, X P, 2007,2010$ and 2013 of this software. The obtained results were the same in all versions.

To ensure the adequacy of tool to its purpose, two researchers from MSc in Production Engineering of UTFPR were asked to test it. Furthermore, two undergraduate students of Analysis and Systems Development of UTFPR checked for errors in its programming logic. The suggestions from the researchers were used during the development of the tool. The identified errors were properly corrected.

To validate the SPSS syntax and tool developed in Microsoft Excel for the calculation of scores and descriptive statistics, simulations were performed with real data applications of the instrument, comparing the results obtained through the tool developed in Microsoft Excel with those obtained by the SPSS syntax. The results returned by software were the same, thus ensuring the reliability of tool in question, such as the SPSS syntax.

\section{THE PROPOSED INSTRUMENT}

\section{Development Of The Instrument}

The instrument developed in this study is named TQWL-42, where the TQWL acronym stands for Total Quality of Work Life, and the suffix 42 refers to the number of QWL-related questions. "Total" in the name of the instrument emphasizes that this evaluates the QWL as a whole, without focusing on a specific aspect.

The TQWL-42 is composed by 47 questions. Five are destined for the knowledge of the sample, while 42 are divided equally into five major spheres: Biological/Physiological, Psychological/Behavioral, Sociological/Relational Economic/Political and 
Environmental/Organizational. These spheres are composed of branches (sub spheres) called aspects, which were clustered in questions.

In an attempt of concatenate a linear thinking to the respondents, it was decided to group together in pairs the areas of QWL that had proximity between them, giving thus rise to the spheres. Each sphere consists of four aspects, each with two questions, adding up to 40 issues, and additionally two questions that focus on self-assessment of QWL from the respondent's viewpoint.

Regarding to questions pertaining each aspect, a pattern was developed, in which a question aims to diagnose the level, in which the variable discussed in aspect is present in the employee's life, while the other question refers to the assessment of the satisfaction level with respect to such variable.

The questions are grouped according to spheres and aspects, so that items pertaining to the same group are answered sequentially. The exception is made only to questions that address the self-assessment of QWL, which are the first and last of the questionnaire. Regardless the grouping by sphere and aspect, there is no mention in the questionnaire that indicates to which sphere or aspect the questions belong.

All questions of TQWL-42 are closed and use a five-item Likert scale (1-5). The extremes represent $0 \%$ and $100 \%$, respectively. The answers scales used are those present in the WHOQOL instruments.

Following the procedure used in the WHOQOL instruments regarding the formulation of questions whose answers scale is inverted, in the TQWL-42 the conversion of the questions that shows such peculiarity is also used. The conversion aims to standardize all the answers of the instrument, so that the more positive the response, the closer it should be to 5 . Therefore, the more negative the response, the more it should approach 1 . Thus, all questions of the instrument are converted to the same answers scale, in which, as a standard, the highest value always represents the best score.

The questions for knowledge of the sample are arranged at the beginning of the questionnaire, followed by questions that contemplate the QWL in the TQWL-42 instrument.

\section{Calculation of scores}

For the scores calculation, the SPSS syntax of TQWL-42 and a tool from Microsoft Excel were developed to perform such calculations.

The proposed tool automatically performs all calculations of scores and descriptive statistics of TQWL-42, and the researchers who use it need only fill the answers given by respondents in specified cells. This tool was developed in order to facilitate the completion and error detection. The logic used by the tool is as follows:

- The cells whose responses are correct (answered with values between 1 and 5) are represented by the fill color green;

- If a respondent has failed to answer nine or more questions (over $80 \%$ of all questions of the instrument), the researcher will be instructed to exclude such respondent. The number of unanswered questions is reported to the researcher and these cells will be highlighted (white fill color);

- If any answer has been filled out with any value that is not included between 1 and 5 , the number of invalid responses will be notified to researcher and invalid responses will be highlighted (red fill color);

- The questions of inverted scale are properly converted;

- The score of the aspects is calculated from the arithmetic mean of the responses of the two questions pertaining to an aspect. If only one of two questions that make an aspect has been answered correctly, the score of the aspect will be the value of the answer to that question. If both questions pertaining to the same aspect are not answered or were answered with a value not listed in the range of 1 to 5 , the score of this aspect is not calculated; 
- The score of the spheres is calculated from the arithmetic mean of the scores of aspects belonging to each sphere. If the score of one aspect has not been calculated, the score of the sphere will be calculated from the arithmetic mean of scores of three not null aspects. The score of the spheres will not be calculated if two or more aspects of the same sphere have not been scored;

- If two or more spheres are not calculated, the exclusion of the respondent of the sample shall be requested;

- It is a calculated "Total" score of the respondent, which is the arithmetic mean of the scores of the 21 aspects of TQWL-42;

- The descriptive statistics of each question, aspect, sphere and "Total" are calculated. The values in the descriptive statistics are: mean, standard deviation, maximum value, minimum value, coefficient of variation and range;

- The mean scores of the aspects and spheres are converted to a scale between 0 and 100 and are displayed in a bar graph.

After the insertion of data in order to use the results, the researcher may copy the individual scores for each respondent, the results of descriptive statistics and graphics. However the researcher cannot change these results. The only area that is allowed the insertion and edition of values is the area of tabulation of the responses of respondents.

The TQWL-42, SPSS syntax and the Microsoft Excel tool for score and descriptive statistics calculation is available online through the URL: http://www.brunopedroso.com.br/tawl42(en).html.

\section{RESULTS ANALYSIS}

In order to analyze the results of TQWL-42, it is suggested to use the QWL scale proposed by Timossi et al. [8], in which a central point (50) characterizes the intermediate level of the QWL, while values below and above this central point ranging between 25 and 75 points are characterized as dissatisfaction and satisfaction, respectively. The values not included in the range 25-75 have their respective trends to total dissatisfaction and total satisfaction, and such total limits are, in theory, non-existent. From this perspective, this classification is expressed in the Figure 1:

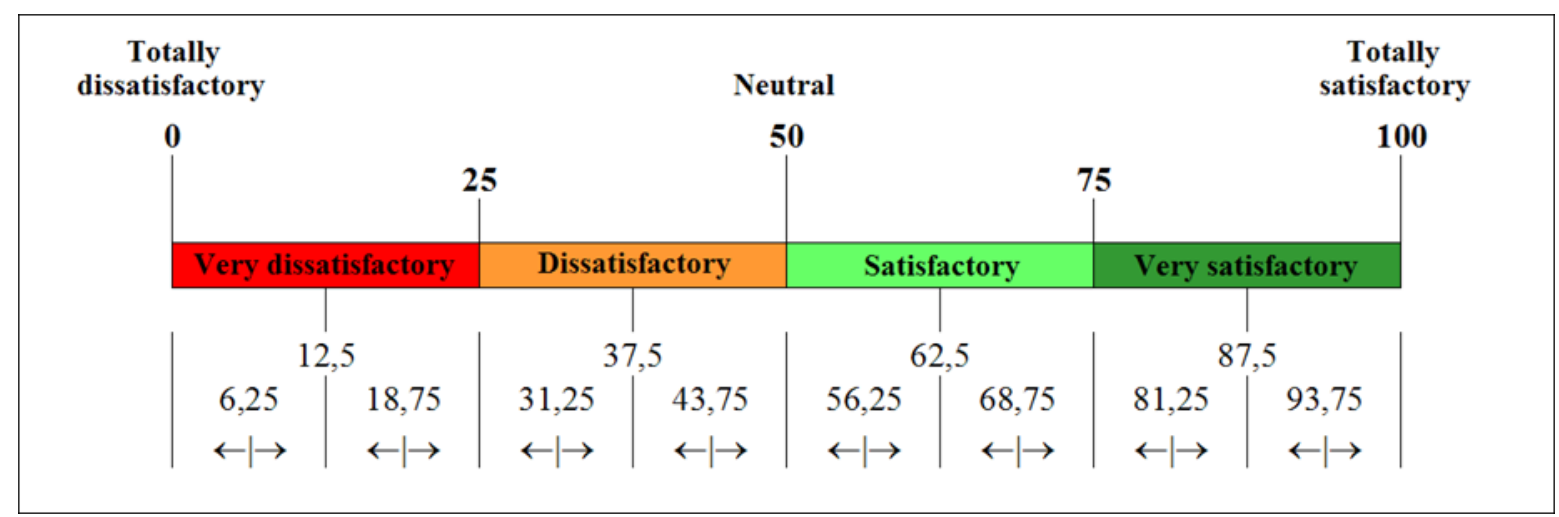

Figure 1. Classification of QWL.

Source: Timossi et al. [8].

Regarding this classification of the QWL it is pertinent to note that, according the authors, the perception of the individual of the QWL is subjective. Quantifying something subjective is possible with the construction of a rational model of thought, which in its pure form does not exist in a reality filled with irrationalities, which is the concrete reality. In this proposal, the anchors and the neutral point describe a model that is considered ideal. Therefore, the results on the scale proposed are neither hypotheses nor propositions, and 
thus cannot be false or true, but valid or non-valid, according to its usefulness for the significant understanding of events studied by the researcher [8].

This classification is not tight and the use of this is merely suggested. At the discretion of the researcher, other scale for the classification of QWL may come to be used.

\section{Verification of Psychometric Properties Sample characteristics}

The characteristics of the sample concerning age, gender, education and marital status are showed on Table 1:

Table 1. Sample characteristics $(n=283)$

\begin{tabular}{ccc}
\hline Variables & $\mathbf{N}$ \\
\hline Age: mean (SD) & $34,3(14,2)$ \\
Gender: women/men & $136 / 147$ \\
Education & & \\
Elementary/middle school & 16 & $(5,65 \%)$ \\
High school & 78 & $(27,56 \%)$ \\
Undergraduate & 103 & $(36,40 \%)$ \\
Graduate & 86 & $(30,39 \%)$ \\
Marital status & & \\
Single & 110 & $(38,87 \%)$ \\
Married/Civil union & 148 & $(52,30 \%)$ \\
Widowed & 03 & $(1,86 \%)$ \\
Separated/Divorced & 22 & $(7,77 \%)$ \\
\hline
\end{tabular}

\section{Internal consistency}

The internal consistency of TWL-42 measured by Cronbach's alpha coefficient can be observed as follows:

Table 2. Cronbach's alpha of TQWL-42 ( $n=283)$

\begin{tabular}{cccc}
\hline Evaluated items & Cronbach's alpha & $\begin{array}{c}\text { Number } \\
\text { of cases }\end{array}$ & $\begin{array}{c}\text { Number of } \\
\text { items }\end{array}$ \\
\hline Biological/Physiological & 0,397 & 278 & 8 \\
Psychological/Behavioral & 0,573 & 278 & 8 \\
Sociological/Relational & 0,672 & 280 & 8 \\
Economic/Political & 0,573 & 281 & 8 \\
Environmental/Organizational & 0,678 & 280 & 8 \\
Aspects & 0,898 & 282 & 21 \\
Spheres & 0,872 & 282 & 5 \\
Spheres + Aspects & 0,930 & 283 & 26 \\
All questions & 0,874 & 265 & 42 \\
\hline
\end{tabular}

\section{Normality test}

Kolmogorov-Smirnov's test returned normal distribution of all the variables showed on Table 2. Therefore, we used Pearson product-moment correlation coefficient for the criterion validity, concurrent validity and test-retest reliability. 


\section{Criterion validity}

The criterion validity performed by Pearson product-moment correlation coefficient of the spheres of TQWL-42 each other returned the following results:

Table 3. Correlation between the spheres of TQWL-42 $(n=283)$

\begin{tabular}{lcccc}
\hline & $\mathbf{1}$ & $\mathbf{2}$ & $\mathbf{3}$ & $\mathbf{4}$ \\
\hline Biological/Physiological (1) & & & & \\
Psychological/Behavioral (2) & 0.534 & & & \\
Sociological/Relational (3) & 0.477 & 0.633 & & \\
Economic/Political (4) & 0.614 & 0.512 & 0.578 & \\
Environmental/Organizational (5) & 0.557 & 0.618 & 0.634 & 0.609 \\
\hline
\end{tabular}

All values significant for $p<0.01$

\section{Concurrent validity}

The Pearson product-moment correlation coefficient between the Total score of TQWL42 and global score of Walton's QWL instrument $(n=76)$ was 0.915 , which was significant for $<0.001$.

\section{Test-retest reliability}

Tables 4 and 5 present the results of test-retest reliability. Table 4 shows the comparative of the mean of spheres' scores:

Table 4. Comparison of scores of spheres between test and retest $(n=61)$

\begin{tabular}{ccc} 
Spheres & \multicolumn{2}{c}{ Mean (SD) } \\
& Test & Retest \\
\hline Biological/Physiological & $3.289(0.52)$ & $3.230(0.58)$ \\
Psychological/Behavioral & $3.629(0.53)$ & $3.545(0.55)$ \\
Sociological/Relational & $3.361(0.55)$ & $3.307(0.54)$ \\
Economic/Political & $3.209(0.56)$ & $3.102(0.60)$ \\
Environmental/Organizational & $3.389(0.69)$ & $3.338(0.62)$ \\
TOTAL & $3.384(0.44)$ & $3.3130 .48)$ \\
\hline
\end{tabular}

Table 5 depicts the Pearson product-moment correlation coefficient between the spheres' scores on test and retest:

Table 5. Pearson product-moment correlation coefficient between test and retest $(n=61)$

\begin{tabular}{cc} 
Spheres & $\begin{array}{c}\text { Pearson product-moment } \\
\text { correlation coefficient }\end{array}$ \\
\hline Biological/Physiological & 0.779 \\
Psychological/Behavioral & 0.837 \\
Sociological/Relational & 0.828 \\
Economic/Political & 0.882 \\
Environmental/Organizational & 0.750 \\
TOTAL All values significant for $p<0.001$
\end{tabular}




\section{DISCUSSION}

The literature about Cronbach's alpha does not establish a reference value to classify the results as consistent or not. A value that has been taken as basis for a questionnaire to be considered consistent is the index higher or equal to 0.70 [10]. Although in all spheres Cronbach's alpha coefficient was lower than 0.70 , there is an inversion when evaluating the values on aspects, spheres and all questions of the instrument, being the lower value 0.866; and taking into account the fact that the instrument is not applied in an attempt to evaluate a single sphere, but the QWL as a whole, we can infer that it has globally a satisfactory internal consistency.

Regarding the criterion validity verified by the Pearson product-moment correlation of TQWL-42 spheres with each other, there were no values out from the other, and in all cases the correlation was significant.

With regard to concurrent validity, the Pearson product-moment correlation between the Total score of TQWL-42 with the overall score of Walton's QWL instrument of 0.915 indicates that there is a good correlation between both instruments.

Concerning the test-retest reliability, we can observe that there was no significant variation in any spheres. The greatest variations were in the Economic/Political $(2.67 \%)$ and the Psychological/Behavioral (2.10\%) spheres. The other spheres and the Total score of the instrument showed a variation lower than $2 \%$. In all spheres, the Pearson product-moment correlation between both applications was significant, indicating that there was stability in the state of the indicators contemplated by TQWL-42 and also in the reapplication of the same.

\section{CONCLUSION}

Although the assessment instruments of QWL in the literature, no validated instrument that assesses the QWL as a whole was recently found, one without focusing on a specific aspect of the work environment.

The development of TQWL-42 was based on the need of consistent approaches with the lifestyles of contemporary society. The subject placed under examination - QWL - had as target the common person who works and lives in the Knowledge Society, which is in constant expansion.

The purpose of validating an instrument that requires little time for its completion and with satisfactory psychometric characteristics was achieved. In addition, a tool in software Microsoft Excel - a widespread application - was designed for the calculation of results of applications of TQWL-42, providing its use without purchasing the software SPSS.

\section{REFERENCES}

1. The WHOQOL Group. WHOQOL user manual. Geneva: World Health Organization; 1998.

2. Pedroso B. Possibilidades e limites da avaliação da qualidade de vida: análise dos instrumentos WHOQOL e modelos clássicos de qualidade de vida no trabalho [thesis]. Campinas (SP): Universidade Estadual de Campinas; 2013

3. Walton RE. Quality of working life: what is it? MIT Sloan Manage Rev. 1973; 15 (1): 11-21

4. Hackman JR, Oldham GR. The job diagnostic survey: an instrument for the diagnosis of jobs and the evaluation of job redesign projects. New Haven: Department of Administrative Sciences of Yale University; 1974.

5. Westley WA. Problems and solutions in the quality of working life. Hum Rel. 1979; 32 (2): 111123.

6. Werther JR, William B, Davis K. Personnel management and human resources. New York: McGraw-Hill; 1981.

7. Nadler DA, Lawler EE. Quality of work life: perspectives and directions. Organ Dyn. 1983; 11 (3): 20-30. 
8. Timossi LS, Pedroso B, Pilatti LA, Francisco AC. Adaptação do modelo de Walton para avaliação da Qualidade de Vida no Trabalho. Rev. Educ. Fís/UEM. 2009; 20 (3): 395-405.

9. Pedroso B, Pilatti LA, Reis DR. Cálculo dos escores e estatística descritiva do WHOQOL-100 utilizando o Microsoft Excel. Rev Bras Qual Vida. 2009;1 (1): 23-32.

10. Cronbach LJ, Meehl PE. Construct validity in psychological tests. Psychol Bull. 1955; 52 (4): 281-302.

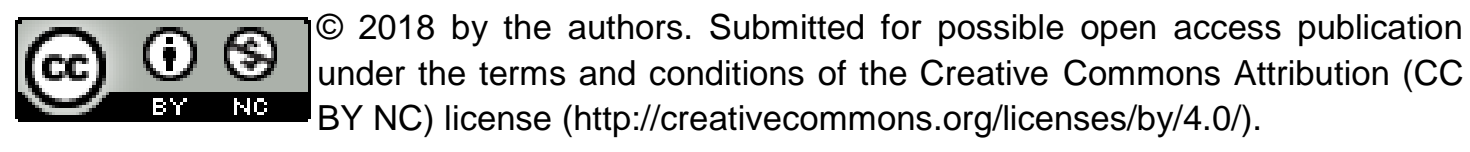

* Correspondence: prof.brunopedroso@gmail.com; Tel.: +55-42-32203141 